\title{
Determination of Glutathione in Biological Material by Flow-Injection Analysis Using an Enzymatic Recycling Reaction
}

\author{
F. A. M. REDEGELD, ${ }^{*, 1}$ M. A. J. VAN OPSTAL, $\dagger$ E. HoudKaMP, ${ }^{*}$ \\ AND W. P. VAN BENNEKOM $\dagger$ \\ *Department of Pharmacology and $\uparrow$ Department of Pharmaceutical Analysis, Faculty of Pharmacy, \\ University of Utrecht, Catharijnesingel 60,3511 GH Utrecht, The Netherlands
}

Received January 7, 1988

\begin{abstract}
A sensitive and specific assay for glutathione using a recycling reaction followed by spectrophotometric detection in a flow-injection analysis system is presented. The proposed method provides specific amplification of the response to glutathione by combined use of the enzyme GSSG reductase and the chromogenic reagent 5,5'-dithiobis(2-nitrobenzoic acid). Both oxidized (GSSG) and reduced (GSH) glutathione are detected, so that GSSG must be determined separately after alkylation of the GSH with $N$-ethylmaleimide. The sensitivity is controlled by the number of times the cycle occurs and therefore by the residence time of the sample in the reactor. This time depends on the reactor length and the flow rate. The influence of residence time, temperature, and enzyme concentration on the response has been studied and the optimum reaction conditions have been selected. The sample throughput is as high as $30 \mathrm{~h}^{-1}$ and the detection limit is 1 pmol GSH at a signal-to-noise ratio of 3 . The method has been evaluated by the quantification of GSH and GSSG in isolated hepatocytes. A high correlation between the new flow-injection analysis method and the original spectrophotometric batch assay has been found (slope $=1.039$, intercept $=0.6, n=216, r=0.977$ ). The main advantages of the proposed method are high sample throughput, high sensitivity, and good reproducibility. () 1988 Academic Press, Inc.

KEY WORDS: glutathione; flow-injection analysis; Ellman's reagent; hepatocytes; enzymatic cycling; $N$-ethylmaleimide.
\end{abstract}

The tripeptide glutathione ( $\gamma$-glutamylcysteinylglycine) is an important sulfhydryl compound present in most organs of various species (1). Glutathione is present predominantly in the reduced form (GSH), whereas only a small amount exists in the oxidized form (GSSG). GSH has an important function in the cellular defense systems, e.g., GSH peroxidases (2) or GSH $S$-transferases (3), against the action of various toxic compounds. Disturbance of the GSH homeostasis could have deleterious effects on cellular processes (4).

Monitoring of the glutathione status, both GSH and GSSG, in toxicological studies is important to gain more insight in the mechanisms of toxicity. Several methods have been

\footnotetext{
${ }^{1}$ To whom reprint requests should be addressed.
}

reported to determine GSH and GSSG in biological materials [for review, see (5)]. Some involve aspecific derivatization of the sulfhydryl group with a fluorescent label or chromophore or use electrochemical detection $(6,7)$. The selectivity is obtained by the use of liquid chromatography.

Other methods are based on the specific assay for glutathione as described by Tietze (8). Glutathione is determined by an enzymatic recycling reaction combined with a chromogenic reaction with 5,5'-dithiobis(2-nitrobenzoic acid) $(\mathrm{DTNB})^{2}$ (Fig. 1). Because the

\footnotetext{
${ }^{2}$ Abbreviations used: DTNB, 5,5'-dithiobis(2-nitrobenzoic acid); TNB, 5-thio-2-nitrobenzoate; NEM, $N$ ethylmaleimide; FIA, flow-injection analysis; SBSR, single bead string reactor; DTT, dithiothreitol; PTFE, polytetrafluoroethylene.
} 


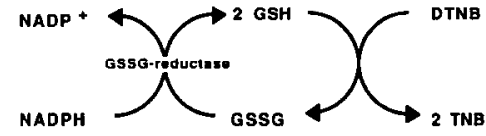

FIG. 1. Recycling reaction of GSII with DTNB, resulting in an accumulation of the thiolate anion TNB, which has a significant absorbance at $412 \mathrm{~nm}(\epsilon=13,600)$.

method responds to both GSH and GSSG, total glutathione (GSH and GSSG) and oxidized glutathione (GSSG) must be determined separately. GSSG can be analyzed separately after alkylating GSH with $N$-ethylmaleimide (NEM). Excess NEM must be removed before analysis because of its inhibiting effect on the GSSG reductase activity. The enzymatic recycling reaction has been applied in a postcolumn reactor in highperformance liquid chromatography by $\mathrm{Al}-$ pert et al. (9).

A sensitive and specific method to determine GSH and GSSG by flow-injection analysis (FIA) is described. The method is based on the recycling reaction of Tietze and allows rapid and simple determination of GSH and GSSG in the picomole range. The flow-injection method is applied to assay of glutathione in biological samples.

\section{MATERIALS AND METHODS}

Reagents. GSSG, NADPH (type X), and glutathione reductase (type III) were obtained from Sigma (St. Louis, MO). GSH was from Merck (Darmstadt, FRG) and DTNB from Fluka (New York). Other reagents were of analytical reagent grade. Millipore water was used for solution preparation.

FIA apparatus. A diagram of the flow-injection manifold is shown in Fig. 2. Carrier and reagents were delivered by a four-channel, variable-speed peristaltic pump (Model IPN4, Ismatec, Switzerland). Sample solutions were introduced into the carrier by using an injector (Model 7125, Rheodyne, CA) provided with a fixed sample loop of $20 \mu 1$. Chemifold type II (Tecator, Sweden) equipped with two Y-pieces was used for premixing the two reagent streams and for mix- ing the combined reagent stream with the carrier stream. The enzymatic recycling reaction was performed in a single bead string reactor (SBSR) of polytetrafluoroethylene (PTFE) tubing ( $2 \mathrm{~m}$ long, $1.5 \mathrm{~mm}$ i.d.) filled with glass beads $(1.0 \mathrm{~mm}$ diameter). The SBSR was fixed in a water bath (Thermomix, Braun Melsungen, FRG) thermostated at $25^{\circ} \mathrm{C}$. The other parts of the flow system were made of 0.5 -mm-i.d. PTFE tubing. The absorbance at $412 \mathrm{~nm}$ was measured with a variable-wavelength flow-through detector (Spectroflow 757 , Kratos, NJ). The fiagrams were recorded on a chart recorder (BD40, Kipp, The Netherlands).

FIA solutions. A $0.4 \mathrm{M}$ potassium phosphate buffer of pH 6.75 was used as carrier and as solvent for reagent solutions R1 and R2. Both reagent solutions were prepared fresh before use and composed as follows: Reagent 1-1 mM DTNB in the carrier; Reagent $2-0.5$ mM NADPH +3 units GSSG-reductase/ml in the carrier. Reagent $\mathrm{l}$ was shielded from light with aluminum foil. Both carrier and rcagent solutions were delivered with a flow rate of $0.2 \mathrm{ml} / \mathrm{min}$ each.

These reagents cannot be stored in one reservoir, since glutathione reductase slowly reduces DTNB (9). The combined reagent solutions were mixed with the carrier solution and delivered at a total flow rate of 0.6 $\mathrm{ml} / \mathrm{min}$ to the reactor.

Standard solutions of both GSH and GSSG in the carrier buffer were prepared fresh before use.

FIA procedure. The influence of the variation of several system parameters on the re-

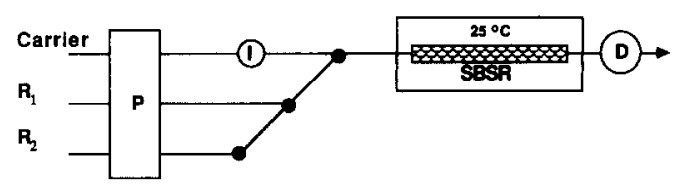

FIG. 2. Manifold for glutathione determination: $P$, pump for propelling carrier and reagents which are pumped at a total flow rate of $0.6 \mathrm{ml} / \mathrm{min}$ through the SBSR; I, sample injector with a 20- $\mu$ l loop; SBSR, singlebead string reactor $(2 \mathrm{~m}$ long, $1.5 \mathrm{~mm}$ i.d.) filled with 1.0 $\mathrm{mm}$ glass beads; $\mathrm{B}$, water bath at $25^{\circ} \mathrm{C} ; \mathrm{D}$, spectrophotometric detector for measuring the absorbance at $412 \mathrm{~nm}$. 
sponse of the flow-injection system was expressed by the amplification factor; this is the ratio of the amplified response to the stoichometric response to GSH. The system will be in the stoichiometric mode by replacing $\mathrm{Re}-$ agent 2 with the buffer solution lacking GSSG reductase.

Under standard conditions the mean residence time was $235 \mathrm{~s}$, the amplification factor was 28 , and the sample throughput could be as high as $30 \mathrm{~h}^{-1}$ without interfering carryover between two successive injected samples. Calibration was performed by using standard solutions of GSSG ranging from 0.0 to $7.5 \mu \mathrm{M}$. The concentrations of total glutathione (GSH plus GSSG) and disulfide (GSSG) were calculated by interpolating the measured peak heights to the calibration curve.

Sample treatment. Perchloric acid (3\% v/v)-treated samples were split. For determination of GSSG, NEM was added to one sample at a final concentration of $10 \mathrm{mM}$.

Excess NEM was destroyed by alkaline hydrolysis, as described recently (10). Some modifications were introduced: a $3 \mathrm{M}$ potassium phosphate ( $\mathrm{pH} 13)$ buffer instead of potassium hydroxide was used to adjust the $\mathrm{pH}$ for alkaline hydrolysis (final $\mathrm{pH}$ 11.2). After 10-15 min the samples were neutralized by addition of $10 \%(\mathrm{v} / \mathrm{v}) \mathrm{HClO}_{4}$.

Centrifugation at $12,000 \mathrm{~g}$ removed precipitated potassium perchlorate. Glutathione determinations were made in the clear supernatant.

For determination of total glutathione the sample was treated in the same way except that an identical volume of water, instead of NEM, was added.

Spectrophotometric determination of glutathione. Glutathione was determined spectrophotometrically according to Tietze (8). The rate of change in absorbance at $412 \mathrm{~nm}$ was monitored with a spectrophotometer (Shimadzu, Japan).

Glutathione was quantified by using a calibration curve of GSSG solutions, treated in the same way as the samples analyzed.
Preparation of rat hepatocytes. Rat hepatocytes were prepared according to Berry and Friend (11), as modified by Groen et al. (12). Hepatocytes (about $1.5 \mathrm{mg} \mathrm{dw} / \mathrm{ml}$ corresponding to $0.5 \times 10^{6}$ cells $/ \mathrm{ml}$ ) were incubated at $37^{\circ} \mathrm{C}$ with different thiol-oxidizing compounds and sampled $(0.4 \mathrm{ml})$ at various times (details are described elsewhere). Cell suspensions were centrifuged gently and, after removal of the supernatants, cells were deproteinized by $0.4 \mathrm{ml} 3 \%(\mathrm{v} / \mathrm{v})$ perchloric acid. All samples were divided into two parts of $200 \mu \mathrm{l}$ and pretreated with $20 \mu \mathrm{l}$ of NEM or $\mathrm{H}_{2} \mathrm{O}$ to determine GSSG and total glutathione concentration, respectively. After treatment of the samples with $66 \mu$ l of potassium phosphate ( $3 \mathrm{M}, \mathrm{pH} 13)$ and neutralization of the samples after 10-15 min with $99 \mu \mathrm{l} 10 \%(\mathrm{v} / \mathrm{v}) \mathrm{HC}_{10}$, glutathione determinations were made in the clear supernatant, using $50 \mu \mathrm{l}$ in the Tietze assay and $20 \mu \mathrm{l}$ in the FIA.

Preparation of rat liver homogenate. Male Wistar rats (Cpb:Wu; 250-300 g) were killed by cervical dislocation. The liver was rapidly removed and stored in cold saline. After the liver was cleared of surrounding tissue and minced, it was homogenized in 2 vol of 150 mM potassium phosphate buffer, $\mathrm{pH} 7.40$, in a Potter-Elvehjem tissue homogenizer. The homogenate was spiked with different concentrations of GSSG after all GSSG-metabolizing enzyme activity was destroyed with perchloric acid ( $3 \% \mathrm{v} / \mathrm{v}$ final concentration). Samples were treated to determine GSSG as described above.

\section{RESULTS AND DISCUSSION}

\section{Sample Preparation}

The simple and rapid method to destroy excess NEM by alkaline hydrolysis according to Sacchetta et al. (10) needs careful $\mathrm{pH}$ adjustments to avoid local extreme alkalization in order to prevent hydrolysis of GSSG. Alkalinization by the use of strong alkaline buffer avoided this problem. It turned out that after alkalinization of the samples a final $\mathrm{pH}$ of 11.2 was reached, allowing the destruction of 


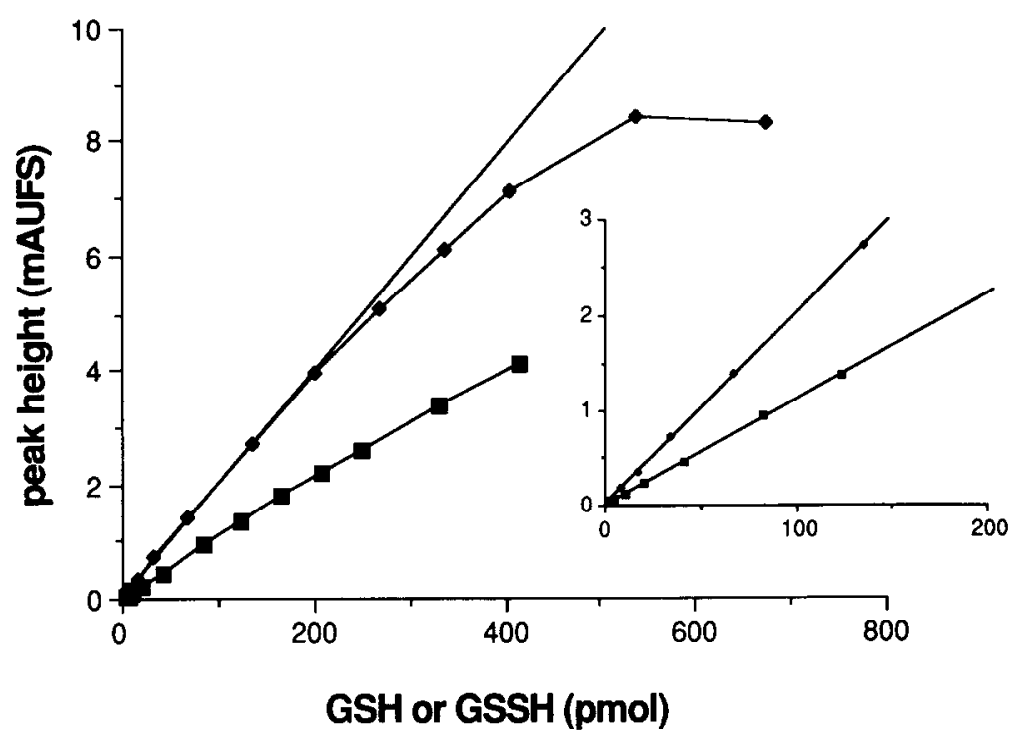

FIG. 3. Typical calibration curves for GSH ( $(\mathbf{)})$ and GSSG $(\bullet)$ determined by FIA. Inset: Linear range of the calibration curves for GSH ( $\square$ ) and GSSG $(\bullet)$ standards.

NEM within $5 \mathrm{~min}$, without affecting the GSSG concentration. This is in agreement with the results of Sacchetta et al. (10). Since the GSSG reductase activity is slightly affected by the presence of residual perchlorate (13) and by the high ionic strength $(9,14)$ of the samples, this would result in an underestimation of the glutathione concentrations of biological samples. Therefore glutathione (GSSG) standard curves have been treated in the same way as the biological samples.

Thus, an alkaline phosphate buffer allows a more rapid and simple removal of excess NEM in biological samples.

\section{Optimal Operating Conditions}

Sensitivity and selectivity of the flow-injection method increase as the residence time increases. The residence time depends on reactor length, reactor diameter, and the flow rate (15). A 2-m-long and 1.5-mm-i.d. SBSR filled with $1.0-\mathrm{mm}$ glass beads was chosen for this system because these reactor dimensions turned out to be a good compromise between the demand for relatively long residence time and the practical requirements of minimal peak broadening and low pressure drop. The residence time can be manipulated further by varying the flow rate through the reactor. The influence of the residence time on the enzymatic recycling reaction was examined in the range $160-600 \mathrm{~s}$. The amplification factor was measured as described under Materials and Methods. The amplification factor increases at increasing residence times. A flow rate of $0.6 \mathrm{ml} / \mathrm{min}$, corresponding to a residence time of $235 \mathrm{~s}$, leading to an amplification factor of 28 , was used in further experiments.

The $\mathrm{pH}$ optimum of the recycling reaction was shown to be dependent on the GSSG reductase concentration (9). Furthermore, the reduction of DTNB by GSSG reductase accelerates with increasing $\mathrm{pH}$, hence increasing the background signal. The use of solutions with a $\mathrm{pH}$ of 6.75 resulted in a first-order response of the recycling reaction with regard to enzyme concentration. At this $\mathrm{pH}$, the background signal was found to be at an acceptable level. To avoid $\mathrm{pH}$ fluctuations in the flow-injection system, the samples were adjusted to $\mathrm{pH} 6$ to 7 .

The reaction of GSH with DTNB shows pseudo-first-order kinetics in excess DTNB 


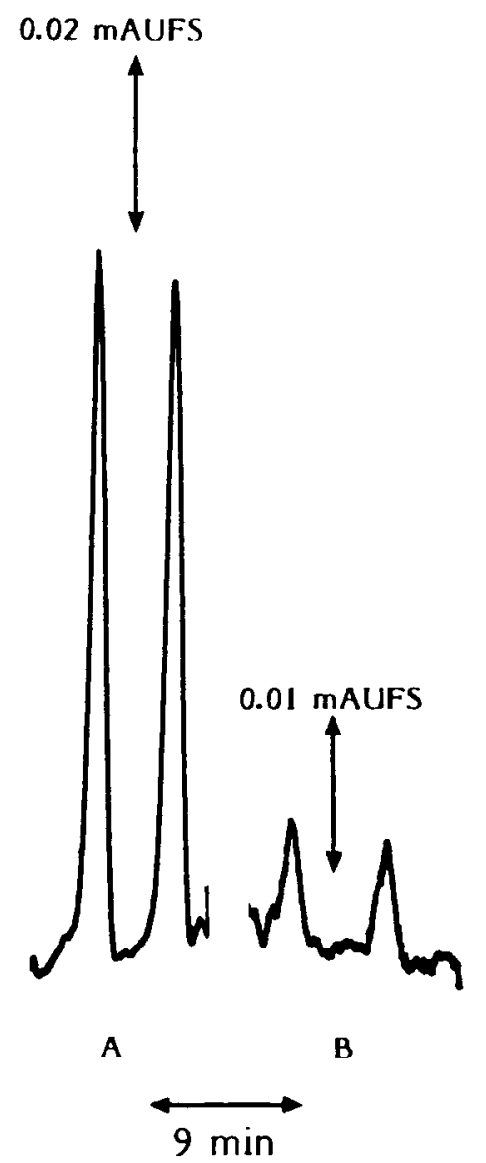

Fig. 4. Typical fiagrams of (A) 10 pmol GSSG and (B) 1 pmol GSSG. Aliquots $(20 \mu \mathrm{l})$ of stock solutions of GSSG $(0.5$ and $0.05 \mu \mathrm{M})$ were subjected to FIA as described under Materials and Methods.

(14). A DTNB concentration of $1 \mathrm{~mm}$ was found to be an excess in the linear range of the recycling reaction; halving the concentration did not affect the linear range of the calibration curve. Additionally, if the concentration of GSSG reductase is combined with an excess of its cofactor NADPH, the enzyme concentration will be the rate-limiting reagent in the recycling reaction (8). The response is proportional to the concentration of GSSG reductase.

Since the rate of the enzymatic reaction accelerates at higher enzyme concentration, the number of recyclings during the residence time increases, resulting in a higher amplification. An increase in the GSSG reductase concentration is accompanied by a simultaneous increase in the background signal due to the reduction of DTNB by the enzyme. A concentration of $3 \mathrm{U} / \mathrm{ml}$ was chosen in subsequent experiments showing a number of recyclings of 28 and acceptable background absorbance. This enzyme concentration was combined with $0.5 \mathrm{mM}$ NADPH which was found to fulfill the condition of being in excess.

For reasons described above excess NEM was destroyed by alkaline hydrolysis with $3 \mathrm{M}$ potassium phosphate buffer. Thereafter, the alkaline samples were neutralized by perchloric acid. However, this treatment produces a relatively high ionic strength in the samples. When these samples were injected into a relatively low carrier buffer concentration, negative peaks were measured due to inhibition of GSSG reductase in the reaction area by the local high ionic strength. A carrier of $0.4 \mathrm{M}$ was chosen to avoid the influence of high ionic strength.

Finally, the influence of temperature on the reaction was investigated. The amplification increases as the temperature increases, due to increased enzyme activity. At $25^{\circ} \mathrm{C}$, an amplification factor of 28 was obtained and further experiments were carried out at this tempcrature.

\section{Calibration}

The linear range of the calibration curve was determined by injecting increasing concentrations of GSH and GSSG. The calibration graphs obtained under the optimized conditions are shown in Fig. 3. The response was linear up to $400 \mathrm{pmol}$ for GSH ( $r$ $=0.9996)$ and up to $200 \mathrm{pmol}$ for GSSG ( $r$ $=0.9997$ ). The intercepts are 0.017 and 0.025 for GSH and GSSG, respectively, and both do not differ significantly from zero ( $\alpha$ $=0.05$ ). The slopes are 10.9 and 20.3 for GSH and GSSG, respectively. Since reduction of one molecule of GSSG generates two molecules GSH, the response of GSSG is twice that of GSH at cquimolar concentrations and consequently the slope of the GSSG 


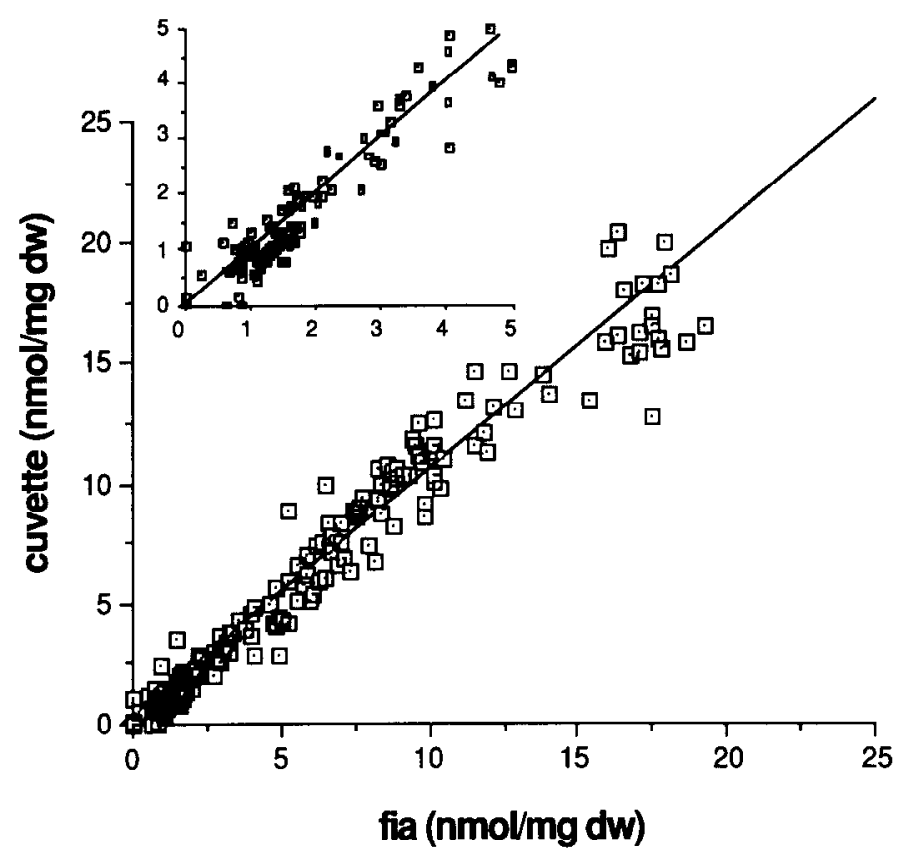

FIG. 5. Correlation between glutathione concentrations in treated hepatocytes, as determined by FIA and by the spectrophotometric method $(r=0.977$, slope $=1.039$, intercept $=0.6, n=216)$. Inset: Correlation at concentrations of glutathione below $5 \mathrm{nmol} / \mathrm{mg}$ dry wt.

curve is twice that of the GSH curve. At higher concentrations the response of GSSG levels off, due to depletion of NADPH. Since in this study only the linear range of the recycling reaction was explored, no further experiments were carried out in the high concentration range. When tissue levels exceeded the linear range levels, samples were diluted to the appropriate concentrations. Alternatively, the linear range can be adjusted by altering the GSSG reductase concentration or the flow rate.

Under reaction conditions optimized for measurements $(\mathrm{pH} 6.75$, residence time 235 $\mathrm{s}$, temperature $25^{\circ} \mathrm{C}$, GSSG reductase $3 \mathrm{U} /$ $\mathrm{ml}$ ) in this study the detection limit (Fig. 4) was found to be 1 pmol GS unit with stock solutions of GSSG. The relative standard deviation for 10 replicatc injections was $2.5 \%$ for $300 \mathrm{pmol}$ and $3.8 \%$ for 18 replicate injections of 20 pmol GSH.

\section{Validation}

To confirm the reliability of the analysis of glutathione by the flow-injection method,
GSH and GSSG concentrations were assayed in hepatocytes. Samples were simultaneously assayed by the flow-injection method and by spectrophotometric assay (8). The correlation $(r=0.977$, slope $=1.039$, intercept $=0.6, n=216)$ between the two methods is shown in Fig. 5 . GSH (16.2 nmol/mg dry wt or $42.6 \mathrm{nmol} / 10^{6}$ cells $)$ and GSSG $(0.4 \mathrm{nmol} /$ mg dry wt or $1.12 \mathrm{nmol} / 10^{6}$ cells) concentrations in untreated hepatocytes were comparable to those reported earlier (16). These results confirm the accurate measurements of either GSH or GSSG by FIA. In addition, the flow-injection method was far more sensitive than the spectrophotometric method.

The efficiency of the procedure was checked by spiking rat liver homogenate with various concentrations of GSSG. The slope of the GSSG calibration curve obtained by the standard addition method (slope $=0.017$, intercept $=1.389$ ) equals that of the standard calibration curve (slope $=0.017$, intercept $=0.019$ ) (Fig. 6), indicating complete recovery of GSSG after sample treatment and analysis. Determination of the GSSG concentra- 


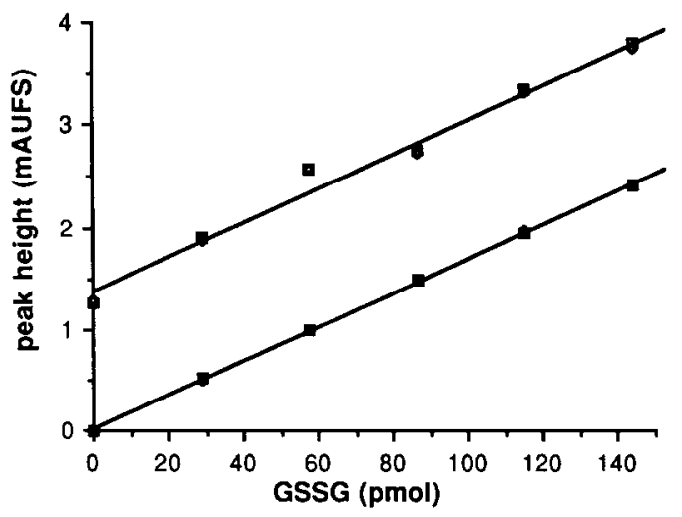

FIG. 6. Standard curve for addition of GSSG to rat liver homogenate, determined by FIA. Upper line $(r=0.997$, slope $=0.017$, intercept $=1.389)$ : standards of GSSG added to the homogenate; lower line ( $r=0.999$, slope $=0.017$, intercept $=0.019$ ): standard calibration curve for GSSG without homogenate. Injection of a 20- $\mu$ l sample corresponds to $2.56 \mathrm{mg}$ liver wet wt.

tion in rat liver by interpolating the intercept to the standard calibration curve resulted in a value $(48.1 \mathrm{nmol} / \mathrm{g}$ wet $\mathrm{wt})$ comparable to earlier values $(19-195 \mathrm{nmol} / \mathrm{g}$ wet $\mathrm{wt})$ $(9,17,18)$.

The specificity of the FIA method can be manipulated by altering the amplification of the reaction. Specificity of the method is governed by the use of GSSG reductase $(14,19,20)$. The recycling reaction amplifies the response to GSH and GSSG over that obtained with the stoichiometric reaction. No amplification of the signal of cysteine, cystine, and dithiothreitol (DTT) was measured. However, the reduced thiol compounds (e.g., cysteine and DTT) reacted with DTNB in a stoichiometric manner. Thus, high concentrations of reduced thiols could interfere in the determination of total glutathione, whereas no interference will be measured in the determination of GSSG.

No interference of endogenous nonglutathione thiols in biological samples will be measured because most of the nonprotein thiol is GSH (1). Furthermore, the recycling reaction of GSH with DTNB amplifies the GSH signal. Only in the case of spiked high concentrations of non-GSH thiols, should interference be checked by determining the am- plification of the signal (in the case of interference the amplification is lower compared with a GSH standard).

In conclusion, FIA offers a rapid, simple, and very sensitive determination of GSH and GSSG in biological samples. Additionally, the flow-injection method is easy to automate. Specificity is obtained by the use of GSSG reductase in the recycling reaction of GSH with DTNB. Sensitivity can easily be manipulated by varying the residence time or the GSSG reductase activity.

\section{REFERENCES}

1. Kosower, N. S., and Kosower, E. M. (1978) Int. Rev. Cytol. 54, 109-160.

2. Wendel, A. (1980) in Enzymatic Basis of Detoxication (Jakoby, W. B., Ed.), pp. 333-353, Academic Press, New York.

3. Boyland, E., and Chasseaud, L. F. (1969) Adv. Enzymol. 32, 173-219.

4. Bellomo, G., and Orrenius, S. (1985) Hepatology 5, 876-882.

5. Jakoby, W. B., and Griffith, O. W. (1987) in Methods in Enzymology, Vol. 143, Academic Press, New York.

6. Lunte, S. M., and Kissinger, P. T. (1985) J. Liq. Chromatogr. 8, 691-706.

7. Debets, A. J. J., Van der Straat, R., Voogt, W. H., Vermeulen, N. P. E., and Frei, R. W. (1988) J. Pharm. Biomed. Anal. 6, 329-336.

8. Tietze, F. (1969) Anal. Biochem. 27, 502-522.

9. Alpert, A. J., and Gilbert, H. F. (1985) Anal. Biochem. 144, 553-562.

10. Sacchetta, P., Di Cola, D., and Federici, G. (1986) Anal. Biochem. 154, 205-208.

11. Berry, M. N., and Friend, D. S. (1969) J. Cell. Biol. 43, 506-520.

12. Groen, A. K., Sips, H. J., Vervoorn, R. C., and Tager, J. M. (1982) Eur. J. Biochem. 122, 87-93.

13. Meister, A., and Anderson, M. E. (1983) Annu. Rev. Biochem. 52, 711-760.

14. Eyer, P., and Podhradsky, D. (1986) Anal. Biochem. 153, 57-66.

15. Reijn, J. M., Van der Linden, W. E., and Poppe, H. (1981) Anal. Chim. Acta 123, 229-237.

16. Eklöw, L., Moldéus, P., and Orrenius, S. (1984) Eur. J. Biochem. 138, 459-463.

17. Stein, A. F., Dills, R. L., and Klaassen, C. D. (1986) J. Chromatogr. 381, 259-270.

18. Davies, M. H., Birt, D. F., and Schnell, R. C. (1984) J. Pharmacol. Methods 12, 191-194.

19. Carlberg, J., and Mannervik, B. (1975) J. Biol. Chem. 250, 5475-5480.

20. Pihl, A., Eldjarn, L., and Bremer, J. (1975) J. Biol. Chem. 227, 339-345. 